Available online at_www.iponlinejournal.com

\title{
Denosumab role in sacral aneurysmal bone cyst: A rare case report
}

\author{
Krishna Subramanyam ${ }^{1}$, Sameer Chaitanya Sahini ${ }^{2 *}$, Satish Kumar ${ }^{3}$ \\ ${ }^{1,2}$ M.S Orthopedics, ${ }^{3}$ DNB Orthopedics, ${ }^{1-3}$ Dept. of Orthopedic, Yashoda hospital, Hyderabad, Telangana, India
}

\begin{abstract}
Aneurysmal bone cyst (ABC) is a benign skeletal tumor which is eccentric, expansile and osteolytic lesion. It contains bloodfilled spaces separated by fibrous septa. Aneurysmal bone cyst occurs most commonly in long bones and rarely in the sacrum. Because of its proximity to sacral nerve roots standard surgical resection or curettage and bone grafting is not optimal. Recent therapeutic strategy is to treat it with denosumab injections subcutaneously. We report a case of aneurysmal bone cyst involved in entire right half of sacrum in a 13 year old girl and we treated with denosumab injections $(60 \mathrm{mg} / \mathrm{ml})$ subcutaneously in every month at regular interval for a period of 12 months and followed for a period of 1 year, there was symptomatic and radiological improvement present.
\end{abstract}

Keywords: Aneurysmal bone cyst (ABC), Bone tumor, Sacrum, Denosumab.

\section{Introduction}

Aneurysmal bone cysts (ABC) are benign bone tumors $^{1}$ with a peak age of incidence in the first 2 decades of life, primarily seen in children and adolescents. $^{2}$ They are located eccentrically in the metaphysis of appendicular bones and axial bones, adjacent to growth plate. ${ }^{3}$ Most common sites are the long bones (67\%), spine (15\%), and pelvis (9\%), although it can involve any part of the skeleton ${ }^{4}$. They usually present as growing mass, swelling with pain and bone destruction; sometimes they are locally aggressive and associated with pathologic fractures. In case of spinal localization, ${ }^{6}$ neurologic deficit may be caused by infiltration and compression of nerve roots. The etiology of the lesion was due to an increase of venous vascular pressure in the bone, resulting in dilation of small vessels that lead to reabsorption of the bone matrix. Recent study reports explain chromosomal rearrangements, such as translocations, resulting in the up regulation of USP6 gene..$^{7-9}$

On radiograph images $^{10}$ (x-ray) and CT scan shows expansive osteolytic lesions, magnetic resonance imaging (MRI) shows septate cystic cavities with blood fluid levels. Histopathologically the lesions are blood-filled cavities separated by fibrous septa not lined by endothelial cells and composed of spindle cells, inflammatory cells, and multinucleated giant cells. Biopsy is mandatory to confirm diagnosis. Treatment options are en bloc resection or curettage followed by bone grafting, sclerotherapy, selective arterial embolization, sclerotherapy or radiotherapy. In spinal and large pelvic aneurysmal bone cysts these treatment options were associated with complications. Recent studies show usage of denosumab in the treatment of aneurysmal bone cysts at inoperable sites. Denosumab $^{5}$ is a human monoclonal antibody that binds the cytokine receptor activator of nuclear factor kappa B ligand (RANKL). RANKL ${ }^{14}$ inhibition blocks osteoclast maturation and function. We report a case of aneurysmal bone cyst involved in the entire right half of sacrum in a 13 year old female child. The patient was treated with denosumab $60 \mathrm{mg} / \mathrm{ml}$ injections at regular monthly intervals for a period of 12 months, and we observed that there is improvement of clinical symptoms and improved radiological findings after 12 months of treatment.

\section{Case Report}

A 13 year old girl presented to us with complaining of low back pain and difficulty while walking since 3 months. Pain increased gradually and aggravated by her daily activities like sitting and sleeping and walking, pain relieved temporarily on taking NSAIDS, there is no history of fever, no history of constitutional symptoms and no history of trauma, family history, and systemic examination were unremarkable. Physical examination was unremarkable except for tenderness over the right sacral area, no motor and sensory deficits, bowel and bladder habits were normal.

Computed tomographic (CT) images revealed a $6.5 \times 3.91 \times 3.7 \mathrm{~cm}$ lytic lesion with thin rim of outer

*Corresponding Author: Sameer Chaitanya Sahini, Dept. of Orthopedic, Yashoda hospital, Hyderabad, Telangana, India

Email: sameerchaitanyasahini@gmail.com

http://doi.org/10.18231/j.ijmpo.2019.024 
cortex in the entire right sacrum (Fig.1a \& 1b). Biopsy shows blood-filled cystic spaces separated by cellular fibrous stroma containing mononuclear cells and rare osteoclast like giant cells. Significant hemosiderin deposition was seen. Because of its presence at inoperable site, routine surgical management ${ }^{4}$ like intra-lesional curettage and bone grafting was not considered. Surgical treatment was associated with high risk of serious permanent morbidity and disability because of proximity to sacral nerves, and so alternate treatments were sought. Denosumab ${ }^{5}$ treatment was started to the patient. Denosumab injection in the dosage of $60 \mathrm{mg} / \mathrm{ml}$ was administered subcutaneously in every month. Vitamin D 400 IU and calcium (500 $\mathrm{mg} /$ day) supplementation was given to the patient to prevent hypocalcemia, ${ }^{11}$ a rare but serious complication of denosumab. After 2 weeks of $1^{\text {st }}$ dosage of denosumab pain was subsided in intensity and her gait also improved and she was able to sleep in any position after 3 weeks of starting treatment. By the end of 1 month, the pain was markedly improved and resolved by 2 months after starting denosumab. A computed tomographic scan at 6 months after denosumab injections showed evidence of new bone formation (Fig. 2a \& 2b) with a more clearly defined cortex. Treatment was continued for 12 months, at which time imaging revealed new bone formation. Calcium, phosphorus, and alkaline phosphatase levels ${ }^{12,13}$ were normal during the treatment, and no major side effects were observed. Biopsy report after treatment revealed new bone formation and hypocellular fibrous stroma that contained scattered mononuclear cells and there was no osteoclast like giant cells. Hemosiderin deposition was rare to absent. Treatment was stopped after 1 year and followed annually.
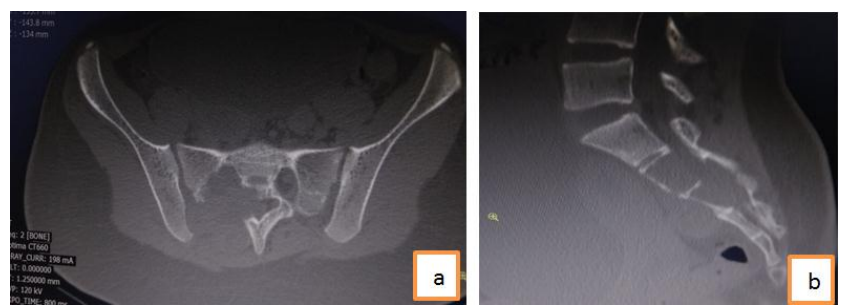

Fig. 1a \& b: Pre-treatment CT scan coronal and sagittal cut images shown lytic area in right half of sacrum.
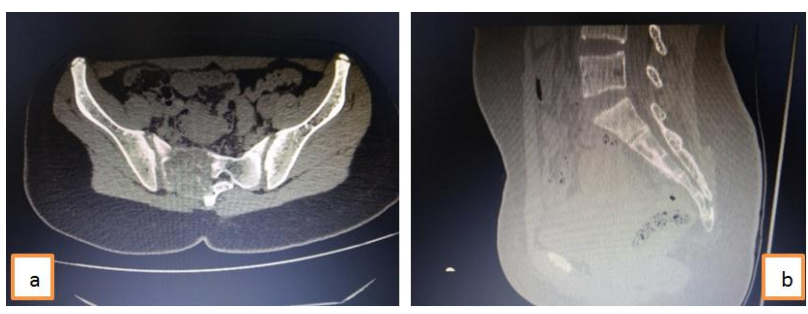

Fig. 2a \& b: post denosumab injections (after 6 months) coronal and sagittal images shows tumor regression and new bone formation.

\section{Discussion}

Aneurysmal bone cysts (ABC) are benign bone tumors. ${ }^{1,2}$ they are locally aggressive and destructive to the bone. They are characterized by blood-filled cavities separated by fibrous septa comprising fibroblasts, inflammatory lympho-histiocytic elements, and multinucleated giant cells ${ }^{3}$. Histopathologically aneurysmal bone cysts contain osteoclast-like mono nuclear giant cells (MNGC) and fibroblast-like cells; this histology picture is similar to giant cell tumors of the bone ${ }^{11}$ (GCT). The giant cells that occur in both giant cell tumors and aneurysmal bone cysts have markers of true osteoclasts, so osteolysis is because of these mononuclear giant cells. RANKL ${ }^{14}$ is highly expressed in stroma of aneurysmal bone cysts and it activates giant cells, and it binds to RANK present on the surface of monocyte and macrophage lineage precursors. The RANK signalling pathway ${ }^{14}$ has vital role in tumor progression. Treatment options available for aneurysmal bone cysts are curettage and bone grafting, embolization, sclerotherapy, and radiotherapy. Surgical management is the gold standard for the treatment of aneurysmal bone cysts $(\mathrm{ABC})$ with a local control rate up to $100 \%$.Other methods has $15-50 \%$ failure rates. Traditional treatment options for spinal ${ }^{6}$ and pelvic aneurysmal bone cysts $(\mathrm{ABC})$ are associated with severe complications, surgical procedures related complications are neurologic impairment, spinal instability or other vital problems. Sclerotherapy ${ }^{20}$ treatment method has response rates up to $85 \%$, after repeated injections. Sclerotherapy ${ }^{21}$ associated complications are local inflammation and thromboembolism. Radiotherapy is now limited use due to the risk of radiation induced sarcomas, vertebral body collapse, and growth arrest in young patients. In consideration of these complications, non-invasive innovative therapies are required to treat the bony lesions.

Denosumab $^{5}$ is a human monoclonal antibody that binds to RANKL and it causes RANKL inhibition blocks osteoclast maturation and function. Denosumab 
is successfully used in the treatment of osteoporosis, skeletal metastases and recently in the treatment of giant cell tumors of bone ${ }^{11}$ (GCTB). The immunohistochemical similarity between giant cell tumor (GCTB) and aneurysmal bone cyst (ABC) justify the hypothesis that denosumab may also have positive effects on aneurysmal bone cyst.

Recent study reports show limited number of patients has been treated by denosumab. Pelle et $\mathrm{al}^{14}$ reported a case of a 5-year-old boy with sacral ABC treated with denosumab $1.2 \mathrm{mg} / \mathrm{kg} / \mathrm{dose}$, and reported there was resolution of pain and neurologic improvement occurred after 2 and 6 weeks of treatment respectively, with a significant reduction of tumor volume; no complications were observed. Skubitz et al $^{15}$ reported a case of a 27 -year-old male with sacral ABC, treated with denosumab 120mg subcutaneously, after 5 months there was resolution of pain and new bone formation on CT scan, decrease in cellularity of fibrous stroma observed. Lange et $\mathrm{al}^{16}$ reported 2 cases of children ( 8 and 11 years old) treated with denosumab $60 \mathrm{mg} / \mathrm{ml}$ for spinal aneurysmal bone cysts at c5 level, where embolization failed, and reported healing of the lesion after 4 months of treatment with improvement of pain, regression of the neurologic deficits and tumor regression. In addition to clinical response, radiological recovery with denosumab treatment was observed in all cases reported in the literature. Palmerini et $\mathrm{al}^{17}$ reported 9 cases treated with denosumab shows decrease of pain and tumor regression with bone formation. Theoretically denosumab usage may be associated with a dosedependent risk to develop osteonecrosis of jaw ${ }^{18}$ $(\mathrm{ONJ})$ in $1.1 \%-2.0 \%$ of patients, and there is a theoretical risk of retardation in linear growth ${ }^{19}$ of children. In all the case reports in the literature showed no such complications were reported. We also observed that there was clinical and radiological improvement in our case after treatment with monthly dosage of $60 \mathrm{mg} / \mathrm{ml}$ denosumab injections subcutaneously for a period of 1 year, there was decrease in pain drastically after 2 weeks, and associated with bone formation on CT scan. During treatment no complications were observed in our patient. But the long-term outcome is unknown. However some questions remain regarding how long to treat and what happens after discontinuation is still requires research.

\section{Conclusion}

We conclude that denosumab treatment in case of aneurysmal bone cysts at inoperable sites can result in symptomatic and radiological improvement and shrinkage of aneurysmal bone cyst size and new bone formation. In view of this, denosumab $(60 \mathrm{mg} / \mathrm{ml})$ is effective in the treatment of sacral aneurysmal bone cysts.

\section{Source of Funding}

None.

\section{Conflict of Interest}

None.

\section{References}

1. Campanacci M, Cervellati C, Donati U. Aneurysmal bone cyst (a study of 127 cases, 72 with long term follows up). Ital J Orthop Traumatol 1976;2:341-53.

2. Cottalorda J, Kohler R, Sales de Gauzy J. Epidemiology of aneurysmal bone cyst in children: a multicentre study and literature review. J Pediatr Orthop B 2004; 13:389-94.

3. Mankin HJ, Hornicek FJ, Ortiz-Cruz E, et al. Aneurysmal bone cyst: a review of 150 patients. J Clin Oncol 2005;23:6756-62.

4. A Shah, P Luthra, AR Mahale, A Kumar, A Venugopal,V Poornima Aneurysmal Bone Cyst Of Sacrum - A Case Report. Ind J Radiol Imag 2006:1:19-21

5. Lange T, Stehling C, Frohlich B. Denosumab: a potential new and innovative treatment option for aneurysmal bone cysts. Eur Spine J 2013; 22:1417-22.

6. Boriani S, Lo SL, Puvanesarajah V. Aneurysmal bone cysts of the spine: treatment options and considerations.

Neurooncology 2014;120:171-8.

7. Oliveira AM, Hsi BL, Weremowicz S. USP6 (Tre2) fusion oncogenes in aneurysmal bone cyst. Cancer Res 2004;64:1920-3.

8. Lau AW, Pringle LM, Quick L. TRE17/ubiquitinspecific protease 6 (USP6) oncogene translocated in aneurysmal bone cyst blocks osteoblastic maturation via an autocrine mechanism involving bone morphogenetic protein dysregulation. J Biol Chem 2010;285:37111-20.

9. Oliveira AM, Chou MM, Perez-Atayde AR, Rosenberg AE. Aneurysmal bone cyst: a neoplasm driven by upregulation of the USP6 oncogene. J Clin Oncol 2006;24 e1; author reply e2.

10. Mark J. Kransdorf and Donald E. Sweet. Aneurysmal bone cyst: Concept, Controversy, Clinical Presentation, and Imaging. AJR 1995;164:573-80.

11. Martin-Broto J, Cleeland CS, Glare PA. Effects of denosumab on pain and analgesic use in giant cell tumor of bone: interim results from a phase II study. Acta Oncol 2014;53:1173-9.

12. Setsu N, Kobayashi E, Asano N, et al. Severe hypercalcemia following denosumab treatment in a juvenile patient. J Bone Miner Metab 2016;34:118-22.

13. Koldkjaer Solling AS, Harslof T, Kaal A, Rejnmark L, Langdahl B. Hypercalcemia after discontinuation of longterm denosumab treatment. Osteoporos Int 2016;27:23836.

14. Pelle DW, Ringler JW, Peacock JD. Targeting receptoractivator of nuclear kappaB ligand in aneurysmal bone cysts: verification of target and therapeutic response. Transl Res 2014;164:139-48. 
15. Skubitz KM, Peltola JC, Santos ER, Cheng EY. Response of aneurysmal bone cyst to denosumab. Spine 2015;40:E1201-E1204.

16. Lange T, Stehling C, Fröhlich B. Denosumab: a potential new and innovative treatment option for aneurysmal bone cyts. Eur Spine J 2013;22:1417-22.

17. Denosumab in patients with aneurysmal bone cysts: A case series with preliminary results Emanuela Palmerini, Pietro Ruggieri, Andrea Angelini, Stefano Boriani, Domenico Campanacci, Giuseppe M. Milano, Tumori J 20181104(5):344-51.

18. Kyrgidis A, Toulis KA. Denosumab-related osteonecrosis of the jaws. Osteoporos Int 2011; 22: 369-370.

19. Wang HD, Boyce AM, Tsai JY. Effects of denosumab treatment and discontinuation on human growth plates. $J$ Clin Endocrinol Metab 2014;99:891-7.
20. Rastogi S, Varshney MK, Trikha V. Treatment of aneurysmal bone cysts with percutaneous sclerotherapy using polidocanol: a review of 72 cases with long-term follow- up. J Bone Joint Surg Br 2006;88:1212-6.

21. Varshney MK, Rastogi S, Khan SA, Trikha V. Is sclerotherapy better than intralesional excision for treating aneurysmal bone cysts? Clin Orthop 2010;468:1649-59.

How to cite this article: Subramanyam K, Sahini SC, Kumar S. Denosumab role in sacral aneurysmal bone cyst: A rare case report. Int J Med Paediatr Oncol 2019;5(3): 